\title{
Acoustic damping of quartz tuning forks in normal and superfluid ${ }^{3} \mathrm{He}$
}

\author{
A. M. Guénault, R. P. Haley, S. Kafanov, M. T. Noble, ${ }^{*}$ G. R. Pickett, M. Poole, \\ R. Schanen, V. Tsepelin $\odot,{ }^{\dagger}$ J. Vonka, ${ }^{\dagger}$ T. Wilcox, and D. E. Zmeev \\ Department of Physics, Lancaster University, Lancaster LA1 4YB, United Kingdom
}

(Received 25 June 2019; revised manuscript received 21 August 2019; published 27 September 2019)

\begin{abstract}
We investigate the damping experienced by quartz tuning fork resonators in normal and superfluid ${ }^{3} \mathrm{He}$ as a function of their resonance frequency from 22 to $250 \mathrm{kHz}$ and contrast it with the behavior of the forks in ${ }^{4} \mathrm{He}$. For our set of tuning forks the low frequency damping in both fluids is well described by the existing hydrodynamic models. We find that the acoustic emission becomes the dominating dissipation mechanism at resonator frequencies exceeding approximately $100 \mathrm{kHz}$. Our results show that the acoustic emission model used in ${ }^{4} \mathrm{He}$ fluid also describes acoustic damping in superfluid ${ }^{3} \mathrm{He}$ and normal ${ }^{3} \mathrm{He}$ at low temperatures using the same geometrical prefactor. The high temperature acoustic damping in normal ${ }^{3} \mathrm{He}$ does not exceed prediction of this model and thus the acoustic damping of moderate frequency devices measured in ${ }^{4} \mathrm{He}$ should be similar or smaller in ${ }^{3} \mathrm{He}$ liquid.
\end{abstract}

DOI: 10.1103/PhysRevB.100.104526

\section{INTRODUCTION}

The interaction of helium fluids with small mechanical resonators has traditionally being studied using vibrating wires, and has led to observations of the quantization of vortices in superfluid ${ }^{4} \mathrm{He}[1,2]$, nucleation of quantum turbulence [3,4], and Landau critical velocity in superfluid ${ }^{3} \mathrm{He}[5]$. Developments in the manufacturing of electronic components and easy access to nanofabrication facilities have brought a plethora of other mechanical devices to helium research, for example quartz tuning forks [6-23], micro and nanoelectromechanical devices (MEMS and NEMS) [24-28], optomechanical resonators [29-31], and carbon nanotubes [32]. Since the 2000s quartz tuning forks have become an established tool to investigate quantum solids [6] and liquids [7-10], where they have been used in studies of the viscosity [7], solubility of ${ }^{4} \mathrm{He}-{ }^{3} \mathrm{He}$ mixtures [10], Andreev retroreflection of quasiparticle excitations in superfluid ${ }^{3} \mathrm{He}[16]$, and in turbulence studies in both helium isotopes [17-21]. The main reasons for the forks' popularity are their high intrinsic quality factor, commercial availability, compact size, and the ease of use. Their working procedures are well documented $[8,11,33]$ and after calibration they can be used as temperature probes $[8,12]$ or pressure gauges $[14,15]$.

In this paper we present the damping behavior of tuning forks in normal and superfluid ${ }^{3} \mathrm{He}$. Our studies show that

\footnotetext{
*t.noble@lancaster.ac.uk

$\dagger$ v.tsepelin@lancaster.ac.uk

¥Present address: Paul Scherrer Institut, WLGA/U119, 5232 Villigen PSI, Switzerland.
}

Published by the American Physical Society under the terms of the Creative Commons Attribution 4.0 International license. Further distribution of this work must maintain attribution to the author(s) and the published article's title, journal citation, and DOI. the acoustic emission of tuning forks in superfluid ${ }^{3} \mathrm{He}$ is virtually identical to that in ${ }^{4} \mathrm{He}$, where it is one of the dominating dissipation mechanisms at low temperatures and high frequencies $[18,22,27]$. While high frequency MEMS and NEMS devices are becoming available for probing superfluid ${ }^{4} \mathrm{He}$ [24-27], so far only low frequency MEMS devices have been successfully operated in liquid ${ }^{3} \mathrm{He}[28,34]$ due to the challenges associated with high normal fluid viscosity and superfluid transition temperature being three orders of magnitude lower, in the low milli-Kelvin regime [35,36]. The NEMS devices are expected to open up a novel regime in studies of superfluid ${ }^{3} \mathrm{He}$ since their dimensions are comparable to the pressure dependent coherence length, which has a range from 20 to $80 \mathrm{~nm}[35,36]$. Based on our results, a study of NEMS resonators in ${ }^{4} \mathrm{He}$ liquid should be sufficient to predict their acoustic damping in superfluid ${ }^{3} \mathrm{He}$ and choose the most sensitive devices. Reducing the dimensions of the cavity surrounding submerged NEMS in superfluid ${ }^{3} \mathrm{He}$ should suppress rising acoustic emission and result in an excellent local detector of thermal excitations [37], which could be used for two-dimensional visualization of topological defects [38]. The fermionic nature of superfluid ${ }^{3} \mathrm{He}$ allows noninvasive detection of existing topological defects via the Andreev reflection of excitations, which sense the changes of the order parameter in the vicinity of the defects $[19,37]$. Furthermore, liquid ${ }^{3} \mathrm{He}$ is a promising environment for cooling electrons in nanosized structures down to a few milli-Kelvin [39]. Hence, understanding damping of submerged NEMS devices may help reach the mechanical ground state using "brute force" cooling, which so far has only been accomplished using much higher frequency systems [40].

\section{EXPERIMENTAL SETUP}

The tuning forks used for our measurements were custom designed and manufactured on quartz wafers of various thicknesses [41]. A wafer contains six individual fork sizes 

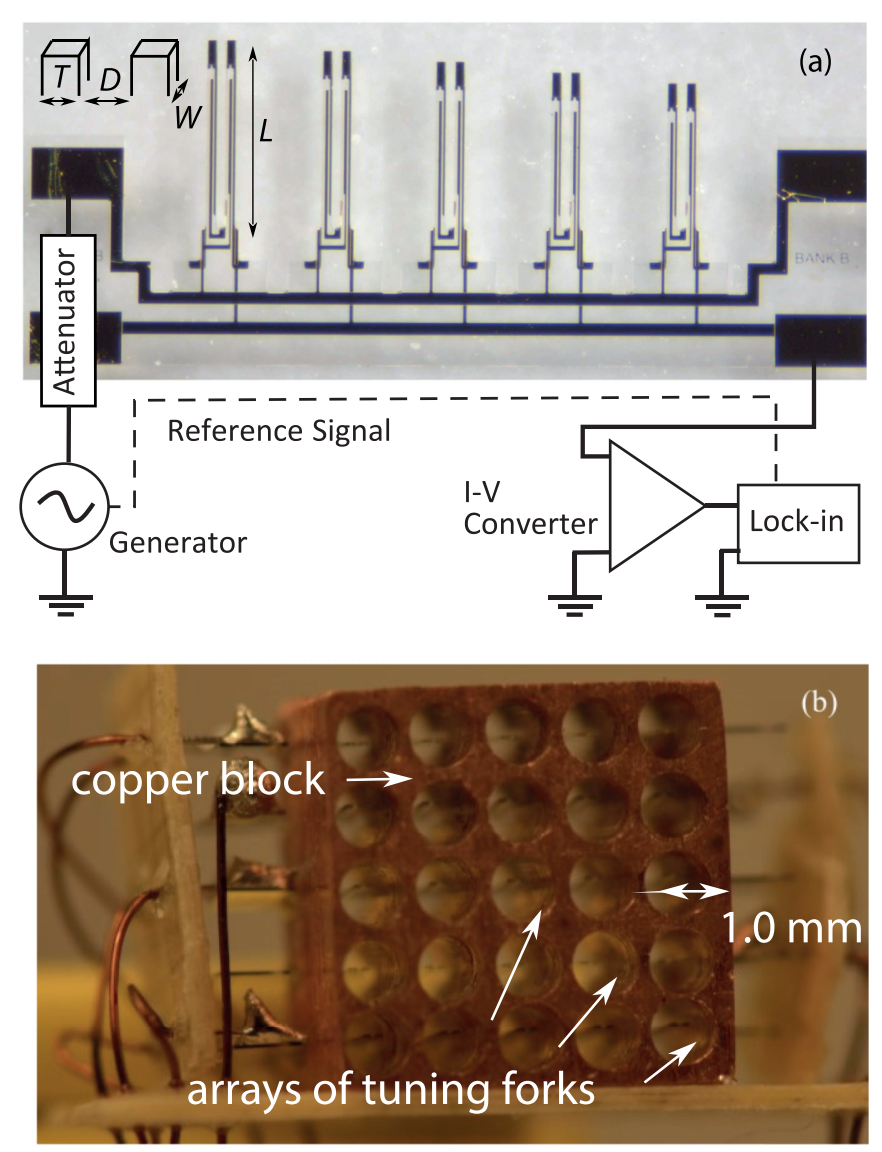

FIG. 1. (a) A schematic of the electronic measurement setup incorporating a photograph of a fork array comprising five tuning forks. (b) A picture of the quasiparticle camera with five tuning fork arrays used in the ${ }^{3} \mathrm{He}$ experiments. Each fork is placed in an individual cylindrical cavity.

and nine five-fork arrays with distinct resonance frequencies. Figure 1(a) shows an example of a tuning fork array. Since all the tuning forks have identical prong thickness $T=90 \mu \mathrm{m}$ and prong separation $D=90 \mu \mathrm{m}$, the resonance frequency of the forks is determined by the prong length $L$. The width of the prong $W$ has no influence on the fork frequency [33] and is determined by the thickness of the wafer. We used two types of forks in our studies $W=50 \mu \mathrm{m}$ and $W=75 \mu \mathrm{m}$. All the forks in an array are connected in parallel, and share drive and signal leads, which significantly reduces the necessary wiring. The tuning fork resonance peak signals do not overlap due to the carefully engineered separation of resonance frequencies and high quality factors that reach $Q \approx 10^{5}$ in vacuum at 4.2 K. Due to a controlled surface finish, our tuning forks offer high reproducibility between different forks and wafers [20-22] compared to commercially available samples, which often show notably different behavior despite a nominally identical geometry.

Figure 1(a) illustrates the principal measurement scheme that we employ to measure tuning forks. Due to the piezoelectric nature of quartz, the forks are voltage driven and produce current as a result of their motion [33]. The generator voltage is attenuated by $80,60,40$, or $20 \mathrm{~dB}$ before entering the cryostat. The signal detected from the fork is amplified by a custom current to voltage converter [42] (transimpedance amplifier) with a gain of $10^{6} \mathrm{~V} \mathrm{~A}^{-1}$ and measured by a lock-in amplifier. For an array of forks adding a summing voltage amplifier, and four pairs of signal generators and lock-in amplifiers, allows us to measure all five forks simultaneously [37].

The ${ }^{3} \mathrm{He}$ measurements were carried out using a nuclear demagnetization stage mounted on an advanced dilution refrigerator [43] and capable of reaching temperatures down to $100 \mu \mathrm{K}$. In our studies we utilized 25 tuning forks, in five arrays, that formed the pixels of a quasiparticle camera [37]. Figure 1(b) shows a photograph of the camera, which is comprised of a copper block of dimensions $5.7 \times 5.7 \times 4.0 \mathrm{~mm}$ and five tuning fork arrays with each fork embedded in an individual cylindrical cavity with a diameter of $1.0 \mathrm{~mm}$. The lengths of the forks in the arrays varied from 1875 to $1400 \mu \mathrm{m}$ and correspond to the frequency range from 22 to $40 \mathrm{kHz}$ for the fundamental resonances. We also utilized the first overtone resonances for each fork, covering a range from 140 to $250 \mathrm{kHz}$ [22]. The sensitivity of mechanical resonators in the ballistic regime of superfluid ${ }^{3} \mathrm{He}$ is inversely proportional to the size of the oscillator, governed by thermal excitations momentum transfer, and hence we chose the thinnest available wafers, $W=50 \mu \mathrm{m}$, to build the camera. Several more traditional vibrating wire viscometers and detectors are placed in the vicinity of the camera and are used for ${ }^{3} \mathrm{He}$ thermometry [44] as well as other experiments. All the resonators described are surrounded by 80 copper sheets necessary for cooling liquid ${ }^{3} \mathrm{He}$ and are a part of the inner cell of a nested Lancaster style experimental cell [45].

The ${ }^{4} \mathrm{He}$ measurements were performed with tuning forks made using $75 \mu \mathrm{m}$ wafers. The low temperature $(450 \mathrm{mK})$ studies were carried out in an experimental cell mounted on a dilution refrigerator [20]. For higher temperature studies [22] arrays and single tuning forks were placed directly in the main bath of a ${ }^{4} \mathrm{He}$ immersion cryostat. The responses of tuning forks embedded in a copper block quasiparticle camera were also carried out in the ${ }^{4} \mathrm{He}$ immersion cryostat. Cooling of the cryostat was achieved by pumping on the helium bath and the ${ }^{4} \mathrm{He}$ temperature was inferred from the saturated vapor pressure [46] measured by a room temperature pressure gauge.

\section{DAMPING OF QUARTZ TUNING FORKS IN HELIUM LIQUIDS}

The damping of tuning forks in helium is a function of temperature $T$, resonance frequency $f$, velocity $v$, and other factors including the detailed confinement of the forks. The total damping (resonance width) of a mechanical resonator $\Delta f_{2}$ in helium is comprised of the intrinsic (or vacuum) contribution $\Delta f_{2}^{\text {int }}$ and the sum of all damping mechanisms supported by the liquid:

$$
\Delta f_{2}=\Delta f_{2}^{\text {int }}+\Delta f_{2}(T)+\Delta f_{2}(v)+\Delta f_{2}(f) .
$$

The intrinsic dissipation of a tuning fork is typically negligible in comparison to the other damping sources $\left(\Delta f_{2}^{\text {int }}<1 \mathrm{~Hz}\right)$, and may become important only in the absence of all other damping sources. For our tuning forks submerged in helium the temperature and frequency dependent damping are the 
largest contributors. They will be introduced first using a framework that permits straightforward comparison with previous measurements of tuning forks in helium $[8,9,22]$.

\section{A. Temperature dependent damping}

The viscosity of a fluid impedes the motion of a mechanical oscillator, and viscous damping is typically described in terms of the Stokes drag. The Stokes contribution in helium superfluids is well understood via the phenomenological twofluid model [36] and is expected to describe experimental observations at temperatures down to about $250 \mu \mathrm{K}$ in ${ }^{3} \mathrm{He}$ [12] and $0.9 \mathrm{~K}$ in ${ }^{4} \mathrm{He}$ [47]. Below these temperatures the mean free path of thermal excitations exceeds the dimensions of the oscillator and the hydrodynamic approach has to be replaced with the ballistic description.

\section{Hydrodynamic damping}

The immersion of a vibrating object into a viscous fluid results in larger damping, detected as an increase in the width of the mechanical resonance and decrease of resonance frequency [48]. Blaauwgeers et al. [8] showed that by using the two-fluid model, the decrease of the resonance frequency in the fluid can be interpreted as an apparent increase of the mass of the forks' prongs due to the fluid backflow around the fork and extra (normal) fluid viscously clamped to the oscillator. The fractional change of the resonance frequency $f_{\mathrm{H}}$ of an object in helium with respect to the vacuum value $f_{0}$ can be expressed as

$$
\left(\frac{f_{0}}{f_{\mathrm{H}}}\right)^{2}=1+\beta \frac{\rho_{\mathrm{H}} V}{m_{\mathrm{e}}}+B \frac{S}{m_{\mathrm{e}}} \sqrt{\frac{\eta \rho_{\mathrm{nf}}}{\pi f_{0}}} .
$$

Here $\beta$ and $B$ are two geometry-dependent parameters of the order of unity, $m_{\mathrm{e}}$ is the effective mass of a tuning fork prong, $V$ and $S$ are the volume and the surface area of a prong, $\rho_{\mathrm{H}}$ is the total density of helium, $\eta$ is helium viscosity, and $\rho_{\mathrm{nf}}$ is the density of the normal fluid component. The coefficient $\beta$ corresponds to the fluid backflow around the fork, while $B$ characterizes the thickness of the normal fluid component clamped to the fork and is governed by the viscous penetration depth $\sqrt{\eta \rho_{\mathrm{nf}} /\left(\pi f_{0}\right)}[8]$.

In the limit where the viscous penetration depth is much smaller than the characteristic size of the oscillator, the width of resonance arising from the viscous damping experienced by the mechanical resonator can be represented via a solution to the Stokes theorem [12]:

$$
\Delta f_{2}^{\text {hyd }}=C \frac{S}{2 m_{\mathrm{e}}} \sqrt{\frac{\rho_{\mathrm{nf}} \eta f_{0}}{\pi}}\left(\frac{f_{\mathrm{H}}}{f_{0}}\right)^{2},
$$

where $C$ is the geometrical factor of the order of unity. After calibration of an oscillator at one known temperature, the coefficient $C$ provides an easy way to determine the temperature of the liquid via the measured resonance frequency and width of the resonance [8]. At high viscosities, such as normal ${ }^{3} \mathrm{He}$ fluid at milli-Kelvin temperatures, a more rigorous approach taking into account slip effects and a large penetration depth is required $[12,49,50]$.

\section{Ballistic damping}

The transition from the hydrodynamic to the ballistic regime can be identified by the disappearance of the resonance frequency change at low temperatures [28,51]. The ballistic damping mechanisms in both ${ }^{4} \mathrm{He}$ and ${ }^{3} \mathrm{He}$ are well understood $[17,50]$ and we will only briefly outline them.

In superfluid ${ }^{4} \mathrm{He}$, the thermal excitations (phonons and rotons) exchanging momentum with the resonator govern its damping, which can be calculated using geometric arguments. The phonon contribution to the resonance width of an oscillating cylinder is given by [50]

$$
\Delta f_{2}^{\mathrm{ph}}=A \frac{k_{\mathrm{B}}^{4}}{45 \hbar^{3} d\left(\rho+\rho_{\mathrm{sf}}\right) c^{4}} T^{4},
$$

where $\rho$ is the density of the resonator material, $\rho_{\mathrm{sf}}$ is the superfluid density, $d$ is the cylinder diameter, and $A$ is a geometrical constant. We can use the fork prong width $W$ as an effective cylinder diameter. We ignore the roton damping contribution as it is negligible compared to the phonons at $450 \mathrm{mK}[50,52]$ where our ballistic ${ }^{4} \mathrm{He}$ measurements were conducted.

In superfluid ${ }^{3} \mathrm{He}$, the oscillator damping arises from its interaction with broken Cooper pairs, the so-called quasiparticles [35,36]. The situation is highly nontrivial due to the presence of Andreev scattering (retroreflection) of the quasiparticles in the superfluid velocity field surrounding a moving object [53]. The net effect of Andreev reflection is an enhancement of the ballistic damping by nearly three orders of magnitude compared to a classical gas with the same excitation density. In the low velocity limit it is possible to approximate the damping width of a vibrating cylinder by

$$
\Delta f_{2}^{\mathrm{qp}}=\frac{d \gamma^{\prime}}{\pi m_{\mathrm{l}}} \frac{p_{\mathrm{F}}^{2}}{k_{\mathrm{B}} T}\left\langle n v_{\mathrm{g}}\right\rangle,
$$

where $\gamma^{\prime}$ is a geometrical constant, $m_{1}$ is the mass per unit length of the cylinder, $p_{\mathrm{F}}$ is the Fermi momentum, and $\left\langle n v_{\mathrm{g}}\right\rangle$ is the thermal quasiparticle flux [17].

\section{B. Frequency dependent damping}

We have already mentioned above that in the ballistic regime the fork damping does not depend on the resonance frequency of the oscillator. According to Eq. (3) the hydrodynamic damping experienced by mechanical oscillators at high temperatures shows a weak, square-root frequency dependence. The square-root frequency dependence is also observed for the nucleation of quantum turbulence in superfluid ${ }^{4} \mathrm{He}$, when the motion of a mechanical oscillator exceeds a certain critical velocity $[17,54]$. The frequency dependence attributed to the emission of sound waves by a tuning fork in ${ }^{3} \mathrm{He}-{ }^{4} \mathrm{He}$ mixtures [7,23,55] and ${ }^{4} \mathrm{He}$ liquid $[22,56,57]$ is nearly an order of magnitude stronger than the square-root dependence. For frequencies above approximately $100 \mathrm{kHz}$ and low tuning fork velocities we expect the acoustic damping to become the main source of dissipation.

Two models for the acoustic emission of a tuning fork have been introduced by the Prague group [18], where the authors have considered spherical and cylindrical emission of sound waves by the prongs of a tuning fork. The spherical 
(“3D”) model seems to describe experimental observations more accurately [22] and predicts the acoustic contribution to the width of the resonance to be

$$
\begin{gathered}
\Delta f_{2}^{3 \mathrm{D}}=C_{3 \mathrm{D}} \frac{\rho_{\mathrm{H}}}{c} \frac{W^{2} L_{e}^{2}}{m_{\mathrm{e}}} \frac{f_{\mathrm{H}}^{4}}{f_{0}^{2}} \Sigma_{3 \mathrm{D}}, \\
\Sigma_{3 \mathrm{D}}=\sum_{\substack{m=0 \\
\text { even }}}^{\infty}(2 m+1) \\
\times\left[j_{m}\left(\frac{\pi f_{\mathrm{H}}(2 T+D)}{c}\right)-j_{m}\left(\frac{\pi f_{\mathrm{H}} D}{c}\right)\right]^{2} .
\end{gathered}
$$

Here $j_{m}$ are spherical Bessel functions, $c$ is the first sound velocity in the liquid, $L_{\mathrm{e}}=0.3915 \mathrm{~L}$ is the effective length of sound wave emittance in the fundamental resonance mode, and $C_{3 \mathrm{D}}$ is a geometrical prefactor of order unity. In the limit where the wavelength of emitted sound is much longer than the relevant fork dimensions, a Taylor expansion of the Bessel functions shows that acoustic damping varies with frequency as $f^{5.5}[18,22]$.

\section{Velocity dependent damping}

The dissipationless motion of superfluids exists only below Landau critical velocity [36], after exceeding which excitations can easily be created. In ${ }^{4} \mathrm{He}$ Landau velocity approaches $\sim 58 \mathrm{~m} \mathrm{~s}^{-1}$ [58] and is unattainable by a macroscopic-size mechanical resonator, due to the creation of turbulence in a liquid impeding the resonator's motion. The production of turbulence by resonators is supported in both normal and superfluid helium and causes significant damping as they lose energy to create vortices in the fluid [17-20]. The critical velocity for the onset of turbulence is typically on the order of $10 \mathrm{~mm} \mathrm{~s}^{-1}$ and is easily achievable by our resonators since their maximum velocity is $\sim 2 \mathrm{~m} \mathrm{~s}^{-1}$.

In superfluid ${ }^{3} \mathrm{He}$ exceeding Landau velocity, which has a value of $\sim 27 \mathrm{~mm} \mathrm{~s}^{-1}$, breaks Cooper pairs, and produces quasiparticle excitations [17]. Quantum turbulence also exists in superfluid ${ }^{3} \mathrm{He}$ and has an onset velocity of the order of several millimeters per second. We avoid turbulence and pair breaking by using velocities far below Landau velocity in ${ }^{3} \mathrm{He}$. All our measurements, in ${ }^{4} \mathrm{He}$ and ${ }^{3} \mathrm{He}$, are carried out at tuning fork velocities below $1 \mathrm{~mm} \mathrm{~s}^{-1}$, which is significantly lower than the expected critical velocities.

\section{RESULTS}

To determine the damping experienced by a tuning fork we sweep the excitation frequency in the vicinity of its resonance and measure the fork's response. We fit the obtained resonance curve with a Lorentzian function to find the resonance frequency $f_{\mathrm{H}}$ and damping width $\Delta f_{2}$ of the fork [22]. First, we will contrast our bulk low temperature measurements in ${ }^{4} \mathrm{He}$ with the results of previous measurements [22] and then introduce measurements carried out in ${ }^{3} \mathrm{He}$.

\section{A. Helium-4 results}

Figure 2 presents the dependence of tuning fork damping as a function of the resonance frequency measured in bulk ${ }^{4} \mathrm{He}$. Below approximately $100 \mathrm{kHz}$ the damping experienced by the forks in helium only weakly changes with their op-

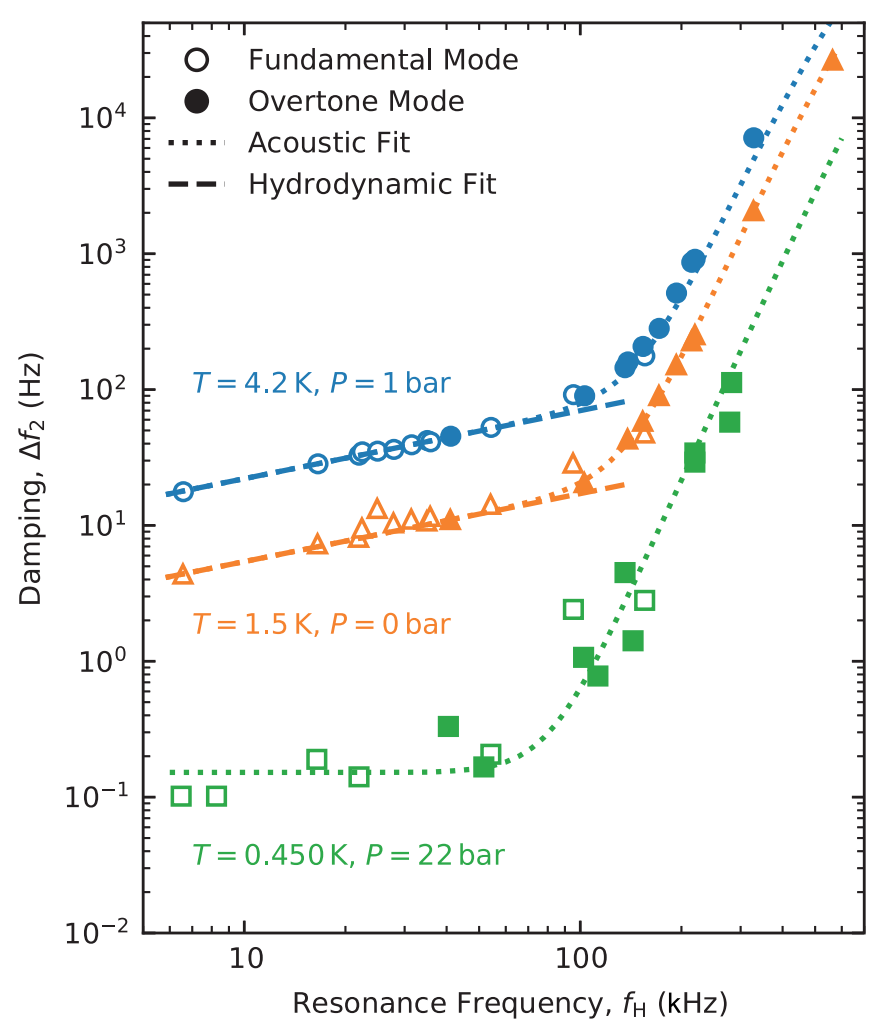

FIG. 2. Log-log plot of frequency dependence of the tuning fork damping in ${ }^{4} \mathrm{He}$ liquid for the fundamental (open symbols) and overtone (filled symbols) modes of the forks. The 4.2 and $1.5 \mathrm{~K}$ data [22] was taken at saturated vapor pressure, while the $450 \mathrm{mK}$ measurements were carried out at a pressure of 22 bars. The dashed line corresponds to the hydrodynamic contribution described by Eq. (3). The dotted lines correspond to the 3D model of acoustic damping with ballistic or hydrodynamic contributions.

erating frequency, but exhibits a significant dependence at higher frequencies. Open and filled symbols correspond to the fundamental mode and the first harmonic of the tuning fork, respectively, and indicate that the frequency dependence observed is not sensitive to the operating mode of the tuning fork. Measurements at temperatures of 4.2 and $1.5 \mathrm{~K}$ at the saturated vapor pressure have been previously reported [22] and show a similar tendency to the low temperature data measured with a different set of tuning forks. The low temperature data was measured at a temperature of $450 \mathrm{mK}$ and a pressure of 22 bars in another experimental cell [20].

The high temperature data at low frequencies is well described within the hydrodynamic framework introduced via Eqs. (2) and (3). The dashed line in Fig. 2 corresponds to fitting parameters $\beta=0.26, B=0.28, C=0.54$ [22]. The dotted line is a combination of the hydrodynamic damping at low frequencies in Eq. (3) and acoustic dominated damping in Eq. (6) at high frequencies with the acoustic emission coefficient $C_{3 \mathrm{D}}=2.17$ [22]. We have used the values of the speed of sound at saturated vapor pressure equal to 190 and $235 \mathrm{~m} \mathrm{~s}^{-1}$ at 4.2 and $1.5 \mathrm{~K}$, respectively [46].

At $450 \mathrm{mK}$ the normal fluid component density of ${ }^{4} \mathrm{He}-$ II is negligible and we expect the data measured at these temperatures to be consistent with the ballistic framework. Hence the low frequency damping should be described by 
phonon interaction and was compared with the predictions of Eq. (4). We obtain a phonon damping constant $A=18$ that significantly exceeds the values reported for vibrating wires [50,52]. The difference could be attributed to the vastly different geometry of the forks compared to vibrating wires and perhaps the nonzero pressure in the experimental cell.

The green dotted line going through the low temperature data in Fig. 2 is a sum of the frequency independent phonon contribution and acoustic damping with coefficient $C_{3 \mathrm{D}}=$ 2.17 used to fit the high temperature data at saturated vapor pressure [22]. We estimated the sound velocity in ${ }^{4} \mathrm{He}$ at 22 bars pressure and $450 \mathrm{mK}$ to be $355 \mathrm{~m} \mathrm{~s}^{-1}$ [59]. It is remarkable that all the acoustic data are described by a single coefficient, despite the data being measured by different forks over a large range of temperatures and pressures. This shows that our custom designed forks manufactured on different wafers are highly reproducible. Turbulent drag measurements carried out in two different laboratories Lancaster and Prague using a set of such forks were also practically identical [21].

\section{B. Helium-3 results}

Prior to introducing ${ }^{3} \mathrm{He}$ into the cell we characterized the intrinsic damping of the tuning forks for the fundamental and first harmonic mode resonances in vacuum at a temperature of $4.2 \mathrm{~K}$. After condensing ${ }^{3} \mathrm{He}$ into the cell, we took measurements during the cryostat cooldown at temperatures of $1.5 \mathrm{~K}$, $115 \mathrm{mK}$, and $10 \mathrm{mK}$. Due to the fermionic nature of ${ }^{3} \mathrm{He}$, its viscosity increases quadratically with decreasing temperature $[12,60]$ and below $10 \mathrm{mK}$ the viscosity of normal ${ }^{3} \mathrm{He}$ becomes comparable to that of olive oil. For our set of tuning forks, which are optimized for superfluid ${ }^{3} \mathrm{He}-\mathrm{B}$ studies, this high viscous damping makes the measurements below $10 \mathrm{mK}$ virtually impossible until the ballistic regime in the superfluid phase is reached. To reach the ballistic regime in superfluid ${ }^{3} \mathrm{He}$ we have precooled the cell in a magnetic field of $6.3 \mathrm{~T}$ to $5 \mathrm{mK}$ using the dilution refrigerator and demagnetized the cell to $50 \mathrm{mT}$, reaching the final temperature of $150 \mu \mathrm{K}$.

The top panel of Fig. 3 shows the dependence of tuning fork damping as a function of the resonance frequency measured in vacuum and in superfluid ${ }^{3} \mathrm{He}-\mathrm{B}$ at $4.2 \mathrm{~K}$ and $150 \mu \mathrm{K}$, respectively. We present both sets of data together since their damping at low frequencies, below $40 \mathrm{kHz}$, is comparable. The tuning fork damping measured in the superfluid contains the intrinsic damping of the fork and hence the liquid contribution is almost identical to the one in vacuum. The vacuum data measured for the first overtone mode lying at frequencies above $100 \mathrm{kHz}$ shows a large degree of scatter (difference in the damping of individual forks). We note that the uncertainty of each data point is negligible, as resonance measured for each fork are stable and reproducible, with the smallest $Q$ factor is of the order of $10^{4}$. We attribute increased damping at certain frequencies to flexing of the base of the tuning fork array and believe that clamping the array base should improve the $Q$ factor further. At moderate frequencies the difference in the damping of individual forks remains large even in the superfluid phase. In some cases the superfluid damping of the forks was smaller than that measured in vacuum, which indicates that the origin of the scatter is likely to be mechanical. The top panel of Fig. 3

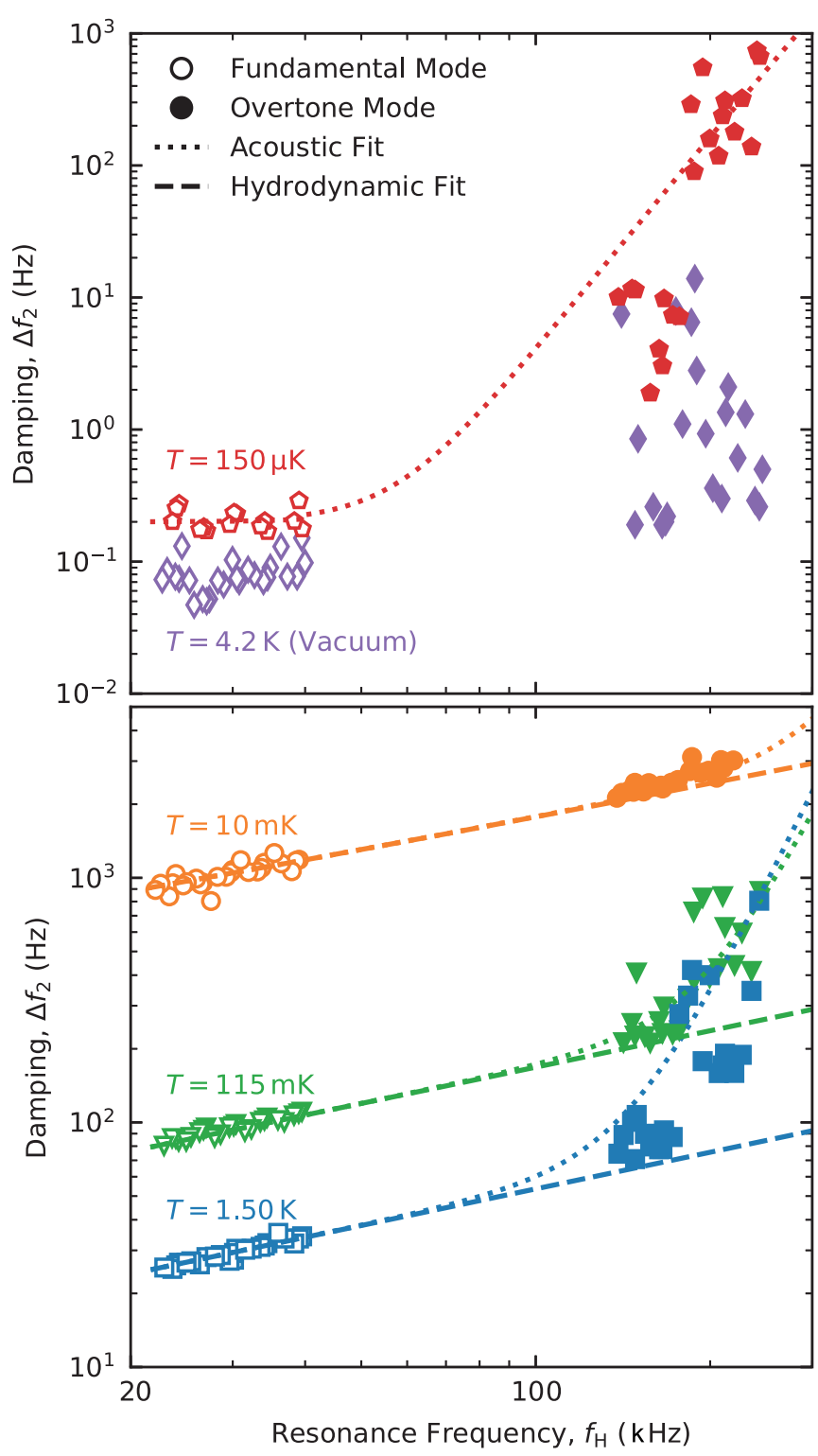

FIG. 3. Log-log plot of the measured damping versus resonance frequency of the forks at the fundamental (open symbols) and overtone modes (filled symbols) in superfluid ${ }^{3} \mathrm{He}-\mathrm{B}$ and vacuum (top); and normal fluid ${ }^{3} \mathrm{He}$ (bottom). The dashed line corresponds to the hydrodynamic damping experienced by the forks. The dotted line shows the acoustic damping model described by Eq. (6) with hydrodynamic or ballistic contributions.

also demonstrates that at the highest operating frequencies the tuning fork damping in the superfluid ${ }^{3} \mathrm{He}$ significantly exceeds the values obtained in vacuum measurements. This indicates the presence of a different damping mechanism compared to the low frequencies.

The measurements taken in normal ${ }^{3} \mathrm{He}$ are presented in the bottom panel of Fig. 3. The fork damping at low frequencies significantly exceeds the intrinsic and the superfluid values. The data show that the damping is larger at the lowest temperatures as expected for a viscous Fermi fluid. The dashed lines in Fig. 3, corresponding to the hydrodynamic damping model, describe the low frequency data well. Due to the high viscosity of ${ }^{3} \mathrm{He}$, instead of the simple framework 
described by Eq. (3) [8] we used a more rigorous calculation in the hydrodynamic regime to account for the large viscous penetration depth. A comparison of the measurements and the hydrodynamic model clearly shows that in the normal fluid the tuning fork resonance widths start to increase above $100 \mathrm{kHz}$ and therefore requires acoustic damping to be taken into account.

The dotted lines in Fig. 3 combine the acoustic contribution described by Eq. (6) with the ballistic damping in the superfluid phase $\left(\gamma^{\prime}=1.6\right.$ to 1.8$)$ and the hydrodynamic damping in the normal fluid (Stokes parameter equal to 1.5), respectively. The combined model seems to reasonably follow the data points in both the superfluid and the normal phases. We chose a value of the acoustic coefficient $C_{3 \mathrm{D}}=2.17$ identical to that in ${ }^{4} \mathrm{He}$ since the forks used differ only in the wafer thickness and $C_{3 \mathrm{D}}$ was constant in the ${ }^{4} \mathrm{He}$ measurements, independent of temperature and pressure changes. The sound velocity in normal ${ }^{3} \mathrm{He}$ liquid at $1.5 \mathrm{~K}$ is $169 \mathrm{~m} \mathrm{~s}^{-1}$ and $184 \mathrm{~m} \mathrm{~s}^{-1}$ at 115 and $10 \mathrm{mK}$, respectively [61]. In the superfluid phase, at our range of temperatures and frequencies zero sound should be emitted instead of first sound [35,36]. Zero sound corresponds to oscillations in the Fermi sphere or the quasiparticle density and has the sound velocity here equal to $190 \mathrm{~m} \mathrm{~s}^{-1}$ [36]. We expect the change from first to zero sound to have little effect on the fork damping as the value of sound velocity hardly changes and the dispersion relations in both modes are identical [36].

The normal $1.5 \mathrm{~K}$ data and the superfluid data plotted in Fig. 3 show that the experimental points seem to deviate significantly from the combined model, and give the impression that the onset of damping happens at high frequencies and exhibits a steep power-law dependence. The observed discrepancy can be explained by considering the shape and the size of the cylindrical cavities surrounding the tuning forks.

\section{DISCUSSION AND CONCLUSIONS}

It is known that cavities suppress acoustic emission $[18,56]$ and while the camera has an open cylinder geometry its effect on the fork's acoustic emission is determined by their relative positions and orientation. To investigate the effect of the cylindrical cavity on emission we placed a fork array in the replica of the camera and measured the damping dependence of the array in a ${ }^{4} \mathrm{He}$ immersion fridge. For the comparison of the data measured at different temperatures in both ${ }^{4} \mathrm{He}$ and ${ }^{3} \mathrm{He}$ it is appropriate to work with the wavelengths of emitted sound rather than the resonance frequencies since the sound velocity varies considerably.

Figure 4 displays the dependence of the tuning fork mechanical resonance width as a function of the sound wavelength for ${ }^{4} \mathrm{He}$ and ${ }^{3} \mathrm{He}$ measurements. The ${ }^{4} \mathrm{He}$ data in the top panel contrasts the bulk measurements [22] shown in Fig. 2 using faded colors against the measurements in the cylindrical cavity for superfluid and normal ${ }^{4} \mathrm{He}$ in bold colors. The dotted curves, corresponding to the combined hydrodynamic and acoustic model, agree well with the bulk data, but fail to describe the cavity data with wavelengths in the range from 1 to $2 \mathrm{~mm}$. These data points are almost entirely described by the hydrodynamic model alone, but still exhibit some

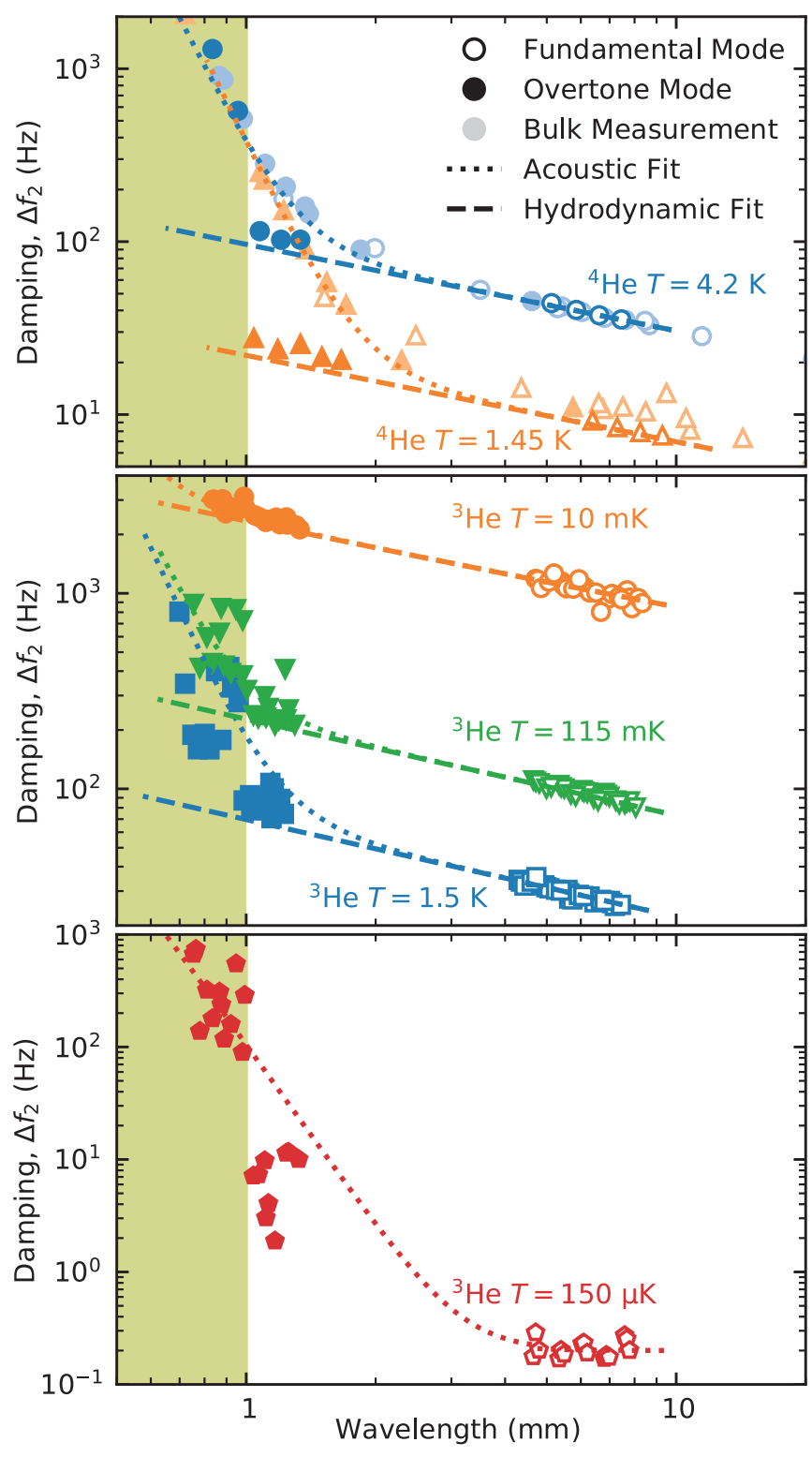

FIG. 4. Log-log plot of the fork damping in ${ }^{4} \mathrm{He}$ (top), normal ${ }^{3} \mathrm{He}$ (center), and superfluid ${ }^{3} \mathrm{He}$ (bottom) versus the sound wavelength corresponding to the fork's fundamental resonance (open symbols) and overtone resonance (filled symbols). The top panel shows bulk measurements in ${ }^{4} \mathrm{He}$ using faded colors along with the measurements performed in a cylindrical cavity in bold colors. The shaded area to the left highlights the wavelengths corresponding to unsuppressed sound emission. The dotted and dashed lines correspond to the total and hydrodynamic damping models, respectively.

degree of the acoustic emission. This suggests that sound emission for wavelengths above about $1 \mathrm{~mm}$ is significantly suppressed, while wavelengths below this threshold show the damping almost in line with what is expected from the bulk ${ }^{4} \mathrm{He}$ measurements. The threshold wavelength of $1 \mathrm{~mm}$ agrees well with the cavity diameter of the camera and the directionality of sound emitted by a tuning fork in the cavity.

The ${ }^{3} \mathrm{He}$ data for normal and superfluid phases shown on the center and bottom panels of Fig. 4 display a trend similar to the ${ }^{4} \mathrm{He}$ measurements. The data points in superfluid ${ }^{3} \mathrm{He}$ 
corresponding to wavelengths in the range from 1 to $2 \mathrm{~mm}$ are neither fully described by the acoustic model nor by the ballistic damping supporting the idea of suppression of sound emission in the surrounding cavity. While the normal ${ }^{3} \mathrm{He}$ results measured at 10 and $115 \mathrm{mK}$ are also consistent with the proposed scenario, the ${ }^{3} \mathrm{He}$ data taken at $1.5 \mathrm{~K}$ show reduced acoustic emission for wavelengths shorter than $1 \mathrm{~mm}$, where sound is expected to be freely emitted. It is not clear what causes this behavior, but it is possible that a combination of imperfect alignment of the arrays in the camera and lower attenuation of the first sound at high temperature [36] affects the acoustic emission of the forks $[18,56]$ and is responsible for the observed differences between the high and low temperature data in ${ }^{3} \mathrm{He}$. High temperature data in normal ${ }^{3} \mathrm{He}$ suggest that the observed acoustic damping does not exceed what is expected from the acoustic emission model. The dilution refrigerator has a poor degree of temperature control in this temperature region and systematic measurements are impractical.

Due to the more complex nature of the fermionic liquid, ${ }^{3} \mathrm{He}$ supports excitations that have no analog in ${ }^{4} \mathrm{He}$. An example of unique ${ }^{3} \mathrm{He}$ excitations are spin waves for which we would expect to have a strong magnetic field dependence [35]. Our measurements in superfluid ${ }^{3} \mathrm{He}$ show that the tuning fork damping is not affected by changing the magnetic field by a factor of 2. It has recently been found that the magnetization of the solid layer of ${ }^{3} \mathrm{He}$ atoms can exert a force on a tuning fork if that fork moves the magnetization vector with respect to the external field in ${ }^{3} \mathrm{He}-\mathrm{B}$ [62]. In our geometry we would expect no force from this effect since the magnetic field and the fork motion are orthogonal to each other. We have also found no sizable changes in normal ${ }^{3} \mathrm{He}$ when magnetic field was changed in the range from 0.1 to $6.3 \mathrm{~T}$.
We conclude that, at low oscillating velocities, the damping experienced by tuning forks in ${ }^{4} \mathrm{He}$ and superfluid ${ }^{3} \mathrm{He}$ liquids can be described by combining the acoustic emission model with the hydrodynamic or ballistic frameworks. The acoustic damping dominates the behavior of tuning forks with resonance frequencies above approximately $100 \mathrm{kHz}$. It is remarkable that sound emission in both isotopes can be described by an acoustic model with a single geometrical coefficient in the normal and superfluid phases for the majority of our tuning forks despite the change of emitted sound mode in ${ }^{3} \mathrm{He}$. Due to the scatter of our high temperature data in normal ${ }^{3} \mathrm{He}$ we can only state that the observed acoustic damping does not exceed what is expected from the acoustic emission model. The latter is useful for predicting properties and behavior of oscillators in superfluid ${ }^{3} \mathrm{He}$, after tests in the much more accessible ${ }^{4} \mathrm{He}$ have been carried out. Our measurements also show that the sound emission can be suppressed by selecting the appropriate cavity size. This should be taken into account for designing experimental apparatus for studies of the onset of quantum turbulence or probing helium excitations using high frequency MEMS and NEMS.

All data used in this paper are available in Ref. [63], including descriptions of the data sets.

\section{ACKNOWLEDGMENTS}

We thank S. M. Holt, A. Stokes, and M. G. Ward for excellent technical support. We thank S. Autti, A. Casey, and V. V. Zavjalov for helpful discussion. This research was supported by the UK EPSRC Grants No. EP/L000016/1, No. EP/I028285/1, No. EP/P024203/1, and No. EP/P022197/1 as well as the European Microkelvin Platform, H2020 Project No. 824109 .
[1] W. F. Vinen, Proc. R. Soc. London Ser. A 260, 218 (1961).

[2] R. J. Zieve, C. M. Frei, and D. L. Wolfson, Phys. Rev. B 86, 174504 (2012).

[3] H. Yano, A. Handa, H. Nakagawa, K. Obara, O. Ishikawa, T. Hata, and M. Nakagawa, J. Low Temp. Phys. 138, 561 (2005).

[4] S. N. Fisher, A. J. Hale, A. M. Guénault, and G. R. Pickett, Phys. Rev. Lett. 86, 244 (2001).

[5] C. A. M. Castelijns, K. F. Coates, A. M. Guénault, S. G. Mussett, and G. R. Pickett, Phys. Rev. Lett. 56, 69 (1986).

[6] S. L. Ahlstrom, D. I. Bradley, M. Človečko, S. N. Fisher, A. M. Guénault, E. A. Guise, R. P. Haley, O. Kolosov, M. Kumar, P. V. E. McClintock, G. R. Pickett, E. Polturak, M. Poole, I. Todoshchenko, V. Tsepelin, and A. J. Woods, J. Low Temp. Phys. 175, 140 (2014).

[7] D. O. Clubb, O. V. L. Buu, R. M. Bowley, R. Nyman, and J. R. Owers-Bradley, J. Low Temp. Phys. 136, 1 (2004).

[8] R. Blaauwgeers, M. Blazkova, M. Človečko, V. B. Eltsov, R. de Graaf, J. Hosio, M. Krusius, D. Schmoranzer, W. Schoepe, L. Skrbek, P. Skyba, R. E. Solntsev, and D. E. Zmeev, J. Low Temp. Phys. 146, 537 (2007).

[9] M. Blažková, M. Človečko, V. B. Eltsov, E. Gažo, R. de Graaf, J. J. Hosio, M. Krusius, D. Schmoranzer, W. Schoepe,
L. Skrbek, P. Skyba, R. E. Solntsev, and W. F. Vinen, J. Low Temp. Phys. 150, 525 (2008).

[10] E. M. Pentti, J. T. Tuoriniemi, A. J. Salmela, and A. P. Sebedash, Phys. Rev. B 78, 064509 (2008).

[11] P. Skyba, J. Low Temp. Phys. 160, 219 (2010).

[12] D. I. Bradley, M. Človečko, S. N. Fisher, D. Garg, A. M. Guénault, E. Guise, R. P. Haley, G. R. Pickett, M. Poole, and V. Tsepelin, J. Low Temp. Phys. 171, 750 (2013).

[13] I. Gritsenko, A. Zadorozhko, V. Chagovets, and G. Sheshin, J. Phys. Conf. Ser. 400, 012068 (2012).

[14] J. Botimer, A. Velasco, and P. Taborek, J. Low Temp. Phys. 186, 93 (2017).

[15] F. M. Huisman, A. E. Velasco, E. Van Cleve, and P. Taborek, J. Low Temp. Phys. 177, 226 (2014).

[16] D. I. Bradley, M. Človečko, E. Gažo, and P. Skyba, J. Low Temp. Phys. 152, 147 (2008).

[17] D. I. Bradley, P. Crookston, S. N. Fisher, A. Ganshin, A. M. Guénault, R. P. Haley, M. J. Jackson, G. R. Pickett, R. Schanen, and V. Tsepelin, J. Low Temp. Phys. 157, 476 (2009).

[18] D. Schmoranzer, M. La Mantia, G. Sheshin, I. Gritsenko, A. Zadorozhko, M. Rotter, and L. Skrbek, J. Low Temp. Phys. 163, 317 (2011). 
[19] J. J. Hosio, V. B. Eltsov, M. Krusius, and J. T. Mäkinen, Phys. Rev. B 85, 224526 (2012).

[20] S. L. Ahlstrom, D. I. Bradley, M. Človečko, S. N. Fisher, A. M. Guénault, E. A. Guise, R. P. Haley, O. Kolosov, P. V. E. McClintock, G. R. Pickett, M. Poole, V. Tsepelin, and A. J. Woods, Phys. Rev. B 89, 014515 (2014).

[21] D. Schmoranzer, M. J. Jackson, Š. Midlik, M. Skyba, J. Bahyl, T. Skokánková, V. Tsepelin, and L. Skrbek, Phys. Rev. B 99, 054511 (2019).

[22] D. I. Bradley, M. Človečko, S. N. Fisher, D. Garg, E. Guise, R. P. Haley, O. Kolosov, G. R. Pickett, V. Tsepelin, D. Schmoranzer, and L. Skrbek, Phys. Rev. B 85, 014501 (2012)

[23] V. A. Bakhvalova, I. A. Gritsenko, E. Ya. Rudavskii, V. K. Chagovets, and G. A. Sheshin, Low Temp. Phys. 41, 502 (2015)

[24] A. Kraus, A. Erbe, and R. H. Blick, Nanotechnology 11, 165 (2000).

[25] D. I. Bradley, R. George, A. M. Guénault, R. P. Haley, S. Kafanov, M. T. Noble, Yu. A. Pashkin, G. R. Pickett, M. Poole, J. R. Prance, M. Sarsby, R. Schanen, V. Tsepelin, T. Wilcox, and D. E. Zmeev, Sci. Rep. 7, 4876 (2017).

[26] T. Kamppinen and V. B. Eltsov, J. Low Temp. Phys. 196, 283 (2019).

[27] K. Y. Fong, D. Jin, M. Poot, A. Bruch, and H. X. Tang, Nano Lett. 19, 3716 (2019).

[28] P. Zheng, W. G. Jiang, C. S. Barquist, Y. Lee, and H. B. Chan, Phys. Rev. Lett 117, 195301 (2016).

[29] L. A. De Lorenzo and K. C. Schwab, New J. Phys. 16, 113020 (2014).

[30] G. I. Harris, D. L. McAuslan, E. Sheridan, Y. Sachkuo, C. Baker, and W. P. Bowen, Nat. Phys. 12, 788 (2016).

[31] A. D. Kashkanova, A. B. Shkarin, C. D. Brown, N. E. FlowersJacobs, L. Childress, S. W. Hoch, L. Hohmann, K. Ott, J. Reichel, and J. G. E. Harris, Nat. Phys. 13, 74 (2017).

[32] A. Noury, J. Vergara-Cruz, P. Morfin, B. Plaçais, M. C. Gordillo, J. Boronat, S. Balibar, and A. Bachtold, Phys. Rev. Lett 122, 165301 (2019).

[33] K. Karraï and R. D. Grober, Ultramicroscopy 61, 197 (1995).

[34] M. Defoort, S. Dufresnes, S. L. Ahlstrom, D. I. Bradley, R. P. Haley, A. M. Guénault, E. A. Guise, G. R. Pickett, M. Poole, A. J. Woods, V. Tsepelin, S. N. Fisher, H. Godfrin, and E. Collin, J. Low Temp. Phys. 183, 284 (2016).

[35] D. Vollhardt and P. Wölfle, The Superfluid Phases of Helium-3 (Taylor and Francis, London, 1990).

[36] C. Enns and S. Hunklinger, Low-Temperature Physics (Springer, Berlin, 2005).

[37] S. L. Alhstrom, D. I. Bradley, S. N. Fisher, A. M. Guénault, E. A. Guise, R. P. Haley, S. Holt, O. Kolosov, P. V. E. McClintock, G. R. Pickett, M. Poole, R. Schanen, V. Tsepelin, and A. J. Woods, J. Low Temp. Phys. 175, 725 (2014).

[38] G. E. Volovik, The Universe in a Helium Droplet (Oxford University Press, Oxford, 2003).
[39] D. I. Bradley, R. E. George, D. Gunnarsson, R. P. Haley, H. Heikkinen, Yu. A. Pashkin, J. Penttilä, J. R. Prance, M. Prunnila, L. Roschier, and M. Sarsby, Nat. Commun. 7, 10455 (2016).

[40] A. D. O'Connell, M. Hofheinz, M. Ansmann, R. C. Bialczak, M. Lenander, E. Lucero, M. Neeley, D. Sank, H. Wang, M. Weides, J. Wenner, J. M. Martinis, and A. N. Cleland, Nature (London) 464, 697 (2010).

[41] Manufactured by the Statek Corporation, 512, N. Main Street, Orange, CA 92868, USA.

[42] S. Holt and P. Skyba, Rev. Sci. Instrum. 83, 064703 (2012).

[43] D. J. Cousins, S. N. Fisher, A. N. Guénault, R. P. Haley, I. E. Miller, G. R. Pickett, G. N. Plenderleith, P. Skyba, P. Y. A. Thibault, and M. G. Ward, J. Low Temp. Phys. 114, 547 (1999).

[44] C. Bäuerle, Yu. M. Bunkov, S. N. Fisher, and H. Godfrin, Phys. Rev. B 57, 14381 (1998).

[45] D. I. Bradley, A. M. Guénault, V. Keith, C. J. Kennedy, I. E. Miller, S. G. Mussett, G. R. Pickett, and W. P. Pratt, Jr., J. Low Temp. Phys. 57, 359 (1984).

[46] R. J. Donnelly and C. F. Barenghi, J. Phys. Chem. Ref. Data 27, 1217 (1998).

[47] M. Blažková, D. Schmoranzer, L. Skrbek, and W. F. Vinen, Phys. Rev. B 79, 054522 (2009).

[48] J. E. Sader, J. Appl. Phys. 84, 64 (1998).

[49] A. M. Guénault, V. Keith, C. J. Kennedy, S. G. Mussett, and G. R. Pickett, J. Low Temp. Phys 62, 511 (1986).

[50] M. Morishita, T. Kuroda, A. Sawada, and T. Satoh, J. Low Temp. Phys. 76, 387 (1989).

[51] T. S. Riekki, J. Rysti, J. T. Mäkinen, A. P. Sebedash, V. B. Eltsov, and J. T. Tuoriniemi, J. Low Temp. Phys. 196, 73 (2019).

[52] A. M. Guénault, A. Guthrie, R. P. Haley, S. Kafanov, Yu. A. Pashkin, G. R. Pickett, M. Poole, R. Schanen, V. Tsepelin, D. E. Zmeev, E. Collin, O. Maillet, and R. Gazizulin, Phys. Rev. B 100, 020506(R) (2019).

[53] S. N. Fisher, A. M. Guénault, C. J. Kennedy, and G. R. Pickett, Phys. Rev. Lett. 63, 2566 (1989).

[54] W. Schoepe, J. Low Temp. Phys. 150, 724 (2008).

[55] E. Pentti, J. Rysti, A. Salmela, A. Sebedash, and J. Tuoriniemi, J. Low Temp. Phys. 165, 132 (2011).

[56] J. Rysti and J. Touriniemi, J. Low Temp. Phys. 177, 133 (2014).

[57] D. Garg, V. B. Efimov, M. Giltrow, P. V. E. McClintock, L. Skrbek, and W. F. Vinen, Phys. Rev. B 85, 144518 (2012).

[58] T. Ellis and P. V. E. McClintock, Philos. Trans. R. Soc. London Sect. A 315, 259 (1985).

[59] B. M. Abraham, Y. Eckstein, J. B. Ketterson, M. Kuchnir, and P. R. Roach, Phys. Rev. A 1, 250 (1970).

[60] M. A. Black, H. E. Hall, and K. Thompson, J. Phys. C Solid State 4, 129 (1971).

[61] H. L. Laquer, S. G. Sydoriak, and T. R. Roberts, Phys. Rev. 113, 417 (1959).

[62] M. Človečko, E. Gažo, M. Skyba, and P. Skyba, Phys. Rev. B 99, 104518 (2019).

[63] http://dx.doi.org/10.17635/lancaster/researchdata/313. 